\title{
ON CHAINS AND POSETS WITHIN THE POWER SET OF A CONTINUUM
}

\section{P. T. MATTHEWS and T. B. M. MCMASTER}

Pure Mathematics Department Queen's University

Belfast BT7 1NN, Northern Ireland

(Received May 6, 1993 and in revised form October 27, 1993)

\begin{abstract}
Transfinite induction is employed to construct a copy of an arbitrary partiallyordered set of cardinality at most $c$ within the power set (quasi-ordered by sub-chain embeddability) of the real line.
\end{abstract}

KEY WORDS AND PHRASES. Partially-ordered set, sub-chain embeddability, real line. 1991 AMS SUBJECT CLASSIFICATION CODES. 06A05, 06A06.

\section{INTRODUCTION.}

One way to explore the structure of a quasi-ordered set $X$ is to seek subsets of it which, under the induced order, are partially- or totally-ordered: for instance the behavior of chains within $X$ is closely related, through a variant of Zorn's lemma, to the existence of elements that are in some sense [4] maximal or minimal in the quasi-order. In her doctoral thesis [2] Matier employed ideas of Stephen Watson to carry out one such investigation on the power set of $\mathbb{R}$ ordered not by set-inclusion but by sub-chain embeddability. She demonstrated that this quasiordered set contains an infinite antichain, and hence deduced that the family of posets on $c$ points or fewer (ordered by sub-poset embeddability) contains an infinite decreasing sequence. This finding has relevance to the behavior of the total negation operation, defined for topological spaces by Bankston [1], when it is applied to partially-ordered topological spaces (see [3] for a brief account).

This note makes use of a modification of the Watson-Matier argument to establish a stronger conclusion about the set of subsets of $\mathbb{R}$; namely, that it contains not only infinite antichains and chains, but also copies of every partially-ordered set whose cardinality does not exceed $c$. An initial examination is also presented of the circumstances (in terms of set-theoretic axioms assumed) in which analogous results may be obtained for higher cardinals.

LEMMA A. Let $C$ be an arbitrary chain, $A$ a non-empty subset of $C$ and $f: A \rightarrow C$ a strictly increasing mapping. If every open interval in $C$ contains a fixed point for $f$ then $f$ is $i d_{A}$.

PROOF. Suppose that there exists $x \in A$ such that $x \neq f(x)$. Then either $x<f(x)$ or $x>f(x)$. In the first case, $y \in(x, f(x))$ implies $x<y$ giving $f(x)<f(y)$, so $f(y) \notin(x, f(x))$ which in turn implies $y \neq f(y)$ : thus $(x, f(x))$ contains no fixed point for $f$. In the second case, $(f(x), x)$ contains no such point.

DEFINITION. Let us call an infinite cardinal $\alpha$ continuum-like if

(i) $\alpha=2^{\beta}$ for some (infinite) $\beta<\alpha$ and 
(ii) there exists a chain $C$ of cardinality $\alpha$ with the following properties:

(a) each open interval in $C$ has cardinality $\alpha$ and

(b) there is a subset $Q$ of $C$ such that card $(Q)=\beta$ and every open interval in $C$ intersects $Q$.

Clearly, $c$ itself is a continuum-like cardinal. We shall address the question of the existence of other continuum-like cardinals later in this article.

Given a strictly increasing function $f: Q \rightarrow C$ (where $Q$ and $C$ are as above) and an element $x$ of $C \backslash Q$, consider the set:

$$
A:=\{\bar{f}(x): \bar{f} \text { is a strictly increasing extension of } f \text { over } Q \cup\{x\}\} .
$$

Whenever this set is a singleton, we shall use the notation $f !(x)$ for its unique element. We make the following definitions:

(i) $\quad x$ is a non-extension point for $f$ if $A=\phi$,

(ii) $x$ is a trivial-extension point for $f$ if $\operatorname{card}(A)=1$ and $f !(x) \in Q$,

(iii) $x$ is a unique-extension point for $f$ if $\operatorname{card}(A)=1$ and $f !(x) \in C \backslash Q$,

(iv) $x$ is a multi-extension point for $f$ if $\operatorname{card}(A)>1$.

It is clear that the four classes of points defined here partition $C \backslash Q$ and we note that there are at most $\beta$ trivial-extension points for $f$ (for otherwise there would exist $x, y$ in $C \backslash Q$ with $x<y$ and $f !(x)=f !(y) \in Q$, contradicting the strictly increasing nature of $f)$. By considering the example $C=\mathbb{R}, Q=\mathbb{Q}, f(x)=x \sqrt{2}$ it is apparent that the number of trivial-extension points can be as high as $\beta$. It can also be as low as zero, as in the case $C=\mathbb{R}, Q=\mathbb{Q}, f(x)=x$. Somewhat less obvious is the observation that the number of multi-extension points is likewise constrained to lie between 0 and $\beta$ :

LEMMA B. Let $\alpha$ be a continuum-like cardinal, $C$ and $Q$ as described in the definition. A given strictly increasing function $f: Q \rightarrow C$ has at most $\beta$ multi-extension points.

PROOF. For each multi-extension point $y$ for $f$ we can choose elements $t_{y}^{1}, t_{y}^{2}$ of $C$ such that $t_{y}^{1}<t_{y}^{2}$ and that

$$
\begin{aligned}
& \bar{f}_{y}^{1}(x)= \begin{cases}f(x) & \text { if } x \in Q, \\
t_{y}^{1} & \text { if } x=y,\end{cases} \\
& \bar{f}_{y}^{2}(x)= \begin{cases}f(x) & \text { if } x \in Q, \\
t_{y}^{2} & \text { if } x=y\end{cases}
\end{aligned}
$$

define two distinct strictly increasing extensions $\bar{f}_{y}^{1}, \bar{f}_{y}^{2}$ of $f$ over $Q \cup\{y\}$. Let $I_{y}$ denote the interval $\left(t_{y}^{1}, t_{y}^{2}\right)$, and note that the family

$$
\left\{I_{y}: y \text { is a multi-extension point for } f\right\}
$$

is pairwise-disjoint: for if $z$ and $y$ are two multi-extension points for $f$ with $z<y$, we can choose $q \in Q$ with $z<q<y$ and observe that for any $a \in I_{z}, b \in I_{y}$ :

$$
\begin{aligned}
a<t_{z}^{2} & =\bar{f}_{z}^{2}(z)<\bar{f}_{z}^{2}(q)=f(q) \\
& =\bar{f}_{y}^{1}(q)<\bar{f}_{y}^{1}(y)=t_{y}^{1}<b
\end{aligned}
$$

so $a \neq b$. Since each of the $I_{y}$ contains a point of $Q$ and $\operatorname{card}(Q)=\beta$, the result follows.

COROLLARY. In the same notation, every open interval in $C$ contains either $\alpha$ nonextension points for $f$ or $\alpha$ unique-extension points for $f$. 
Let $\mathscr{P}_{Q}(C)$ denote the set of all those subsets of $C$ which contain $Q$ and consider it as a quasi-ordered set (qoset) under subchain embeddability: that is, given $A, B \in \mathcal{P}_{Q}(C)$ we write $A \leq B$ if and only if $A$ is order-isomorphic to a subset of $B$ (where $A$ and $B$ inherit the order on $C)$.

THEOREM. Let $S$ be a given partially-ordered set of cardinality $\alpha$. There is a subset of $\mathcal{P}_{Q}(C)$ which is isomorphic to $S$.

PROOF. Denote by $\mathcal{F}$ the set of strictly increasing functions from $Q$ into $C$. Since $\operatorname{card}(\mathcal{F}) \leq \alpha^{\beta}=\alpha, \mathcal{F} \times S$ has cardinality $\alpha$ and can be expressed as the range of an $\alpha$-sequence:

$$
\mathcal{F} \times S=\left\{\left(f_{t}, s_{t}\right): t \in \alpha\right\}
$$

where we are viewing $\alpha$ as an ordinal. Make an arbitrary choice of $q_{0} \in Q$. Transfinite induction will now serve to construct three $\alpha$-sequences $\left(x_{\delta}, \delta \in \alpha\right),\left(y_{\delta}, \delta \in \alpha\right),\left(z_{\delta}, \delta \in \alpha\right)$ in the set $(C \backslash Q) \cup\left\{q_{0}\right\}$.

Let $\gamma \in \alpha$ and suppose that we have already chosen, for each $\delta<\gamma$ in $\alpha$, elements $x_{\delta}, y_{\delta}, z_{\delta}$ of $C$ such that

(i) $x_{\delta}, y_{\delta} \in(C \backslash Q) \cup\left\{q_{0}\right\}, z_{\delta} \in C \backslash Q$,

(ii) all choices are distinct except for repetitions of $q_{0}$,

(iii) whenever $f_{\delta}=i d_{Q}$ then $x_{\delta}=y_{\delta}=q_{0}$,

(iv) whenever $f_{\delta} \neq i d_{Q}$ then

etther $x_{\delta}$ is a unique-extension point for $f_{\delta}$ and $y_{\delta}=f_{\delta} !\left(x_{\delta}\right)$

or $x_{\delta}$ is a non-extension point for $f_{\delta}$ and $y_{\delta}=q_{0}$.

Now if $f_{\gamma}=i d_{Q}$ choose $x_{\gamma}=q_{0}, y_{\gamma}=q_{0}$ and, bearing in mind that the cardinality of $C \backslash Q$ exceeds that of the set of all previously-made choices, select $z_{\gamma}$ in $C \backslash Q$ distinct from all the $x_{\delta}, y_{\delta}$ and $z_{\delta}$ for $\delta<\gamma$. On the other hand, suppose $f_{\gamma} \neq \imath d_{Q}$. If $f_{\gamma}$ possesses $\alpha$ non-extension points then choose one which is different from all preceding choices, denoting it by $x_{\gamma}$, put $y_{\gamma}=q_{0}$ and assign to $z_{\gamma}$ any value in $C \backslash Q$ distinct from all previous selections. If not, then $f_{\gamma}$ must have a strictly increasing extension $f_{\gamma}^{*}$ over a subset $D$ of $C$ such that $C \backslash D$ has cardinality less than $\alpha$; since each interval in $C$ has $\alpha$ elements, this $D$ will therefore be order-dense. An appeal to Lemma A and the Corollary guarantees the existence of an open interval $J_{\gamma}$ in $C$ which is free from fixed points of $f_{\gamma}^{*}$ and contains $\alpha$ unique-extension points for $f_{\gamma}$. Once again, since fewer than $\alpha$ points have previously been identified we can select one of these $\alpha$ unique-extension points $x_{\gamma}$ in such a way that $x_{\gamma}$ and $f_{\gamma} !\left(x_{\gamma}\right)$ differ from all preceding choices, and note that $f_{\gamma} !\left(x_{\gamma}\right) \neq x_{\gamma}$ since $x_{\gamma} \in J_{\gamma}$; pick also $z_{\gamma} \in C \backslash Q$ distinct from all other chosen elements. This completes the inductive step, and we are accordingly assured of the existence of $\alpha$-sequences $\left(x_{\delta}\right),\left(y_{\delta}\right),\left(z_{\delta}\right)$ satisfying the above conditions (i) to (iv) for every $\delta$ in $\alpha$.

For each $s$ in the poset $S$ (order denoted by $\leq$ ) we now define

$$
I_{s}=\left\{x_{\delta}, z_{\delta}: s_{\delta} \leq s\right\}
$$

It is immediate from the definition that $r \leq s$ implies $I_{r} \subseteq I_{s}$ and therefore $Q \cup I_{r}$ trivially embeddable into $Q \cup I_{s}$.

Supposing now that $r \not s$ in $S$, consider the hypothesis that $Q \cup I_{r}$ could be embedded in $Q \cup I_{s}$. Then we could find a strictly increasing function

$$
j: Q \cup I_{r} \rightarrow Q \cup I_{s}
$$


The pair $(\jmath \mid Q, r)$ belongs to $\mathscr{F} \times S$ and is therefore listed as $\left(f_{\delta}, s_{\delta}\right)$ for some $\delta \in \alpha$. Two possibilities must be considered.

(I) $\left.J\right|_{Q}=\imath d_{Q}$. Here Lemma $\mathrm{A}$ implies that $J$ is the identity map on $Q \cup I_{r}$, giving $Q \cup I_{r} \subseteq Q \cup I_{s}$. Yet since $s_{\delta}=r \not \cdots z_{\phi} \in I_{r}$ but $z_{\delta} \notin I$, yielding a contradiction.

(II) $\left.J\right|_{Q} \neq \imath d_{Q}$. This time. $x_{\phi}$ will be either a unique-extension point or a non-extension point for $f_{\delta}$. In the first case. since $x_{\delta} \in I_{r}$ and $\left.J\right|_{Q \cup\left\{x_{\delta}\right\}}$ is strictly increasing.

$$
\jmath\left(x_{\delta}\right)=\left.\jmath\right|_{Q \cup\left\{x_{\delta}\right\}}\left(x_{\delta}\right)=f_{\delta} !\left(x_{\delta}\right)=y_{\delta}
$$

forces $y_{\delta}$ to belong to $Q \cup I_{s}$, contrary to the definitions. In the second, no strictly increasing extension of $\left.j\right|_{Q}$ over $Q \cup\left\{x_{\delta}\right\}$ could exist: and yet, as we saw in the discussion of the first case, $\left.\jmath\right|_{Q \cup\left\{x_{\delta}\right\}}$ is such an extension.

We conclude that, when $r \not s$, no order-embedding of $Q \cup I_{r}$ into $Q \cup I_{s}$ can be obtained. Thus the map

$$
\theta: S \rightarrow \Phi_{Q}(C)
$$

defined by $\theta(s)=Q \cup I_{s}$ satisfies the condition

$$
r \leq s \text { if and only if } \theta(r) \leq \theta(s)
$$

and establishes an order-isomorphism between $S$ and the sub-poset $\theta(S)$ of the qoset $\mathscr{P}_{Q}(C)$.

COROLLARY 1. Given any poset $S$ with $\operatorname{card}(S) \leq \alpha$, we can find a subset of $\mathcal{P}_{Q}(C)$ which is isomorphic to $S$.

PROOF. Extend $S$ in any fashion to yield a poset $S^{*}$ of cardinality $\alpha$. By the theorem there is an embedding

$$
\theta: S^{*} \rightarrow \theta\left(S^{*}\right) \subseteq \Phi_{Q}(C) .
$$

The restriction of $\theta$ to $S$ now embeds the latter into $\mathscr{P}_{Q}(C)$ as required.

COROLLARY 2. Any poset of cardinality not exceeding $c$ can be embedded in $\mathscr{P}_{\mathbf{Q}}(\mathbb{R})$.

NOTE. The question of which infinite cardinals are continuum-like appears difficult to resolve fully, and will certainly depend to some extent on the axiom system adopted. For instance, if we assume the negation of the continuum hypothesis $(C H)$, so that $\aleph_{1}<c$, it will evidently be impossible to express $\aleph_{1}$ in the form $2^{\beta}$, whence $\aleph_{1}$ will not be continuum-like: this contrasts with its status when $C H$ itself is assumed. One positive result is fairly easy to obtain: it will follow from the generalized continuum hypothesis $(G C H)$ that every successor cardinal $\alpha$ is continuum-like. For let $\beta$ be the immediate cardinal predecessor of $\alpha$, so that $G C H$ implies $\alpha=2^{\beta}$, and define

$$
A=\{\text { all } \beta \text {-sequences of } 0 \text { s and } 1 \text { s that are ultimately constant at } 0\} .
$$

Then

$$
\begin{aligned}
\operatorname{card}(A) & \leq \sum_{\delta<\beta} 2^{\delta} \leq \sum_{\delta<\beta} \beta[\text { using } G C H \text { again }] \\
& =\beta^{2}=\beta<\alpha .
\end{aligned}
$$

Next put $C=\{$ all $\beta$-sequences of 0 s and $1 \mathrm{~s}\} \backslash A$ and impose on $C$ the lexicographic ordering, converting it into a chain with $\alpha$ elements.

Let $x=\left(x_{\gamma}, \gamma \in \beta\right)$ and $y=\left(y_{\gamma}, \gamma \in \beta\right)$ be any elements of $C$ for which $x<y$. Then there 
must exist $\delta<\beta$ for which $x_{\delta}=0$ and $y_{\delta}=1$; let $\zeta$ denote the least cardinal for which $y_{\zeta}=1$ and $\zeta>\delta$ (recalling that $y \notin A)$. Now any $z=\left(z_{\gamma}, \gamma \in 3\right)$ for which

$$
\begin{aligned}
& z_{\gamma}=y_{\gamma} \quad \text { if } \gamma<\zeta, \\
& z_{\zeta}=0
\end{aligned}
$$

will lie in the open interval $(x, y)$ of $C$. There are $2^{\beta}$ such sequences $z$, so every interval in $C$ has cardinality $\alpha$ as required.

Now if $Q=\{$ all $\beta$-sequences of 0 s and 1 s that are ultimately constant at 1$\}$ we again have $\operatorname{card}(Q) \leq \beta$; indeed, equality occurs here since it is easy to exhibit $\beta$ distinct elements of $Q$ (those consisting simply of a 'block' of 0 s followed by a 'block' of 1s). In the previous paragraph, the $z$ constructed to lie between $x$ and $y$ could have been chosen to have $z_{\eta}=1$ for all $\eta>\zeta$, therefore belonging to $Q$ : this verifies that $Q$ is order-dense in $C$ and concludes the demonstration.

\section{REFERENCES}

1. BANKSTON, P., The total negation of a topological property, Illinois J. Math. 23 (1979), 241-252.

2. MATIER, J., Total negation in general topology and in ordered topological spaces, Ph.D. thesis, Queen's University Belfast, 1991.

3. MATIER, J. and McMASTER, T.B.M., Iteration of the 'anti' operation in ordered topological spaces and in other categorical contexts, Boll. Un. Mat. Ital., to appear.

4. MATTHEWS, P.T., and McMASTER, T.B.M., A viewpoint on minimality in topology, Bull. Irish Math. Soc., submitted. 


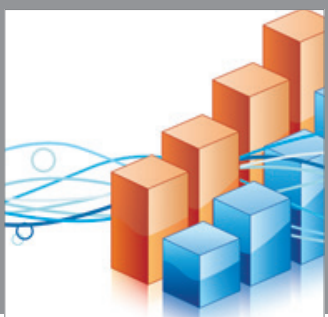

Advances in

Operations Research

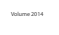

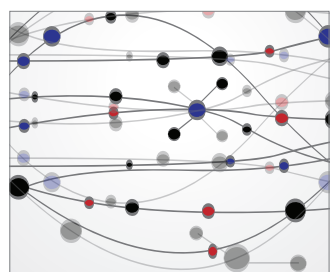

\section{The Scientific} World Journal
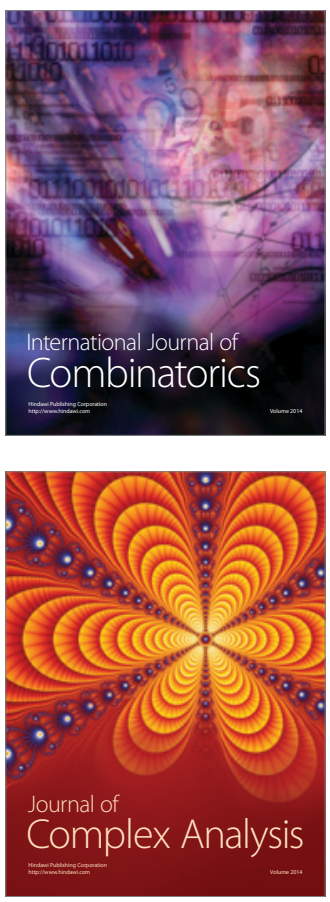

International Journal of

Mathematics and

Mathematical

Sciences
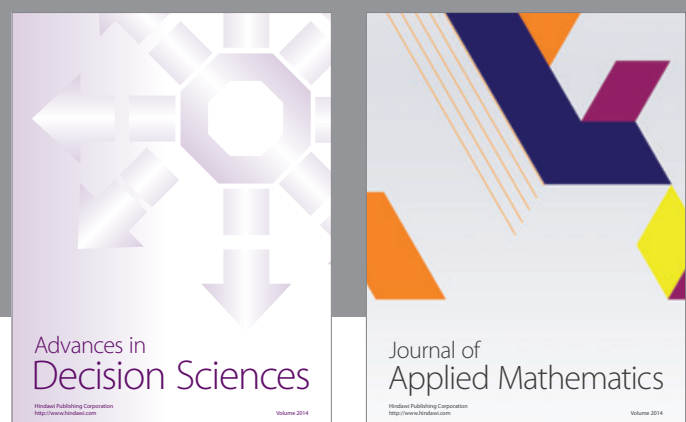

Journal of

Applied Mathematics
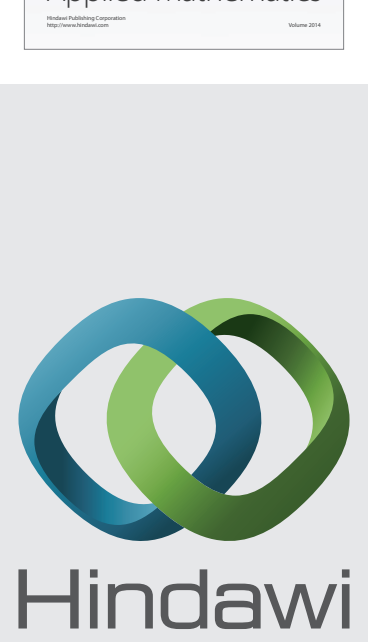

Submit your manuscripts at http://www.hindawi.com
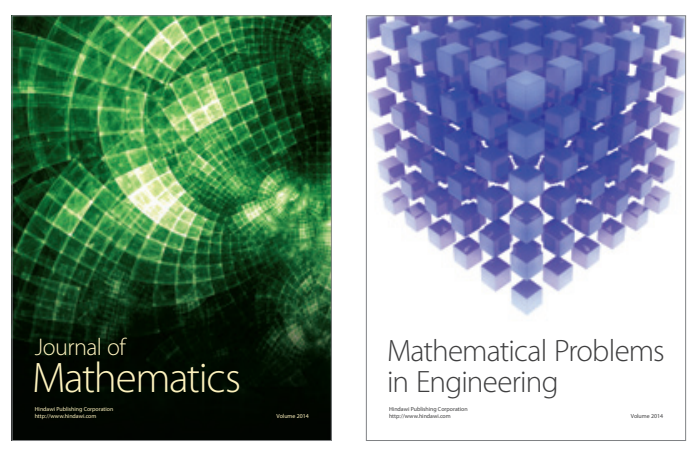

Mathematical Problems in Engineering
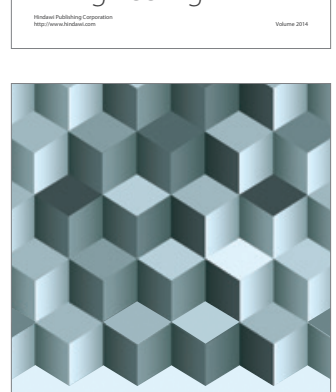

Journal of

Function Spaces
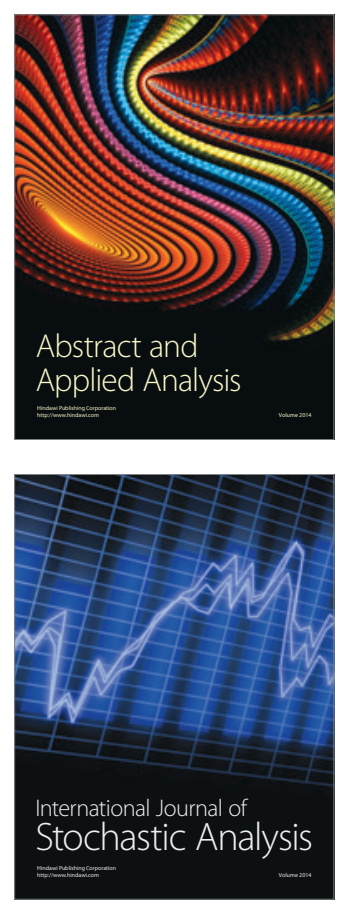

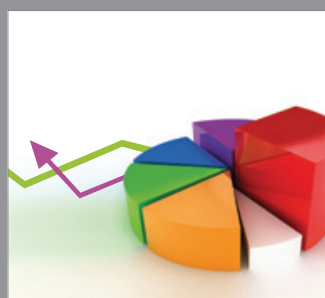

ournal of

Probability and Statistics

Promensencen
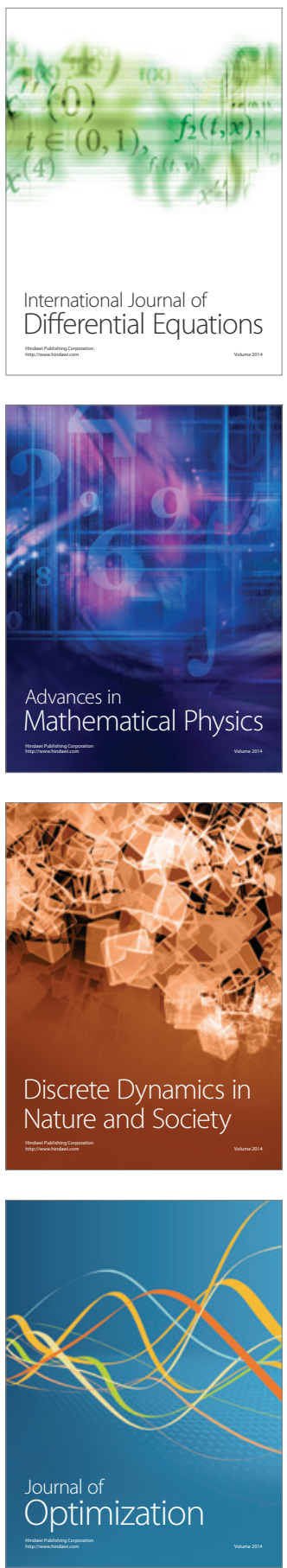